\title{
La ditícil tarea de articular historia, filosofía e introducción a la física. Una perspectiva americana
}

\section{James T. Cushing \\ $R x$}

Curiosamente, la enseñanza de la física más superficialmente empirista y menos «conceptualmente cargada», menos «filosófica», ha coexistido con un planteamiento selectivo de la materia: los alumnos que no la comprendan quedarán en el camino bacia la universidad o las carreras «cientificas». El desdén por buscar vias bistórico-filosóficas para que la física sea comprendida por todos parece de hecho haber logrado que tampoco sea comprendida por sus elegidos. Desde esta tesis, este artículo aborda la utilidad del enfoque bistórico-filosófico en la enseñanza de la física.

Un curso de introducción a las ciencias para todos los alumnos, sean de ciencias o no, debe lograr un equilibrio entre tres metas: (1) Dar al alumno la capacidad de explicar los fenómenos sensibles del mundo; (2) Dar al alumno una apreciación de la naturaleza corregible del conocimiento humano, incluido el conocimiento científico; y (3) Desarrollar a varios niveles, según el destinatario, una capacidad técnica en la aplicación de las leyes de la disciplina a casos particulares. Cursos típicos para alumnos que no son de ciencias se concentran en la primera meta y dedican bastante menos esfuerzo a la segunda y a la tercera, mientras que cursos para alumnos de ciencias se concentran casi exclusivamente en la tercera, en la capacidad técnica del alumno, con poca atención, si la hay, a su apreciación de la naturaleza del conocimiento. La meta que normalmente se olvida en ambos casos es la segunda, en la que incluyo la Historia y la Filosofía de la ciencia. Por tanto estoy a favor de un papel central de la Historia y la Filosofía de la ciencia en cualquier curso de introducción a la Física. Las observaciones y comentarios que hago en este artículo están basados en veinte años de experiencia enseñando cursos de Física desde el nivel introductorio al de licenciado así como de crítica de manuscritos para varias editoriales y contactos con otros críticos. 


\section{RACIONALIDAD DE LA HISTORIA Y LA FILOSOFIA EN UN CURSO DE CIENCIAS}

Hasta los más pragmáticamente orientados de entre nosotros concederían sin duda importancia en nuestra sociedad basada en la tecnología y la ciencia a tener cierta comprensión de, y familiaridad con, la ciencia. Sin embargo, puede ocurrir que no se admita que la Historia y la Filosofía de la ciencia es importante para el alumno de ciencias ya que se supone que el dominio técnico de la materia que pide el curso tradicional de Ciencias, Ingeniería o Física mostrará al alumno qué es la ciencia a través de convertirlo en aprendiz en prácticas de la disciplina. Pero, naturalmente, cualquiera que haya practicado las ciencias sabe por experiencia que la ciencia es más de lo que se presenta en los libros de texto. No sólo deben adquirirse técnicas adicionales con la práctica, sino que en la ciencia es más importante la cuestión del juicio. Esto es esencial, especialmente para cualquiera que trabaje en el límite del desarrollo de la ciencia donde se vuelve dolorosamente evidente que no existen algoritmos para el descubrimiento y construcción de leyes y teorías científicas, ni siquiera para el juicio y selección de teorías ya propuestas. Un estudio razonablemente fiel y filosóficamente sensible de episodios pasados de la Historia de la Física da al alumno cierta visión y apreciación de la dificultad y de la falta de certeza absoluta que acompaña a la construcción del conocimiento científico. Esto se contrapone absolutamente a las versiones «comprimidas», torpemente ahistóricas, del descubrimiento y el progreso que se encuentran normalmente en textos de introducción a la Física. Pocos alumnos de ciencias son conscientes de la similitud de los aspectos creativos del descubrimiento científico y las artès, esto es, la ciencia es un modo de conocer, pero no uno que sea intrínsecamente cierto o cualitativamente distinto de otros modos humanos de conocer.

Una vez que un alumno se ha sensibilizado hacia las presuposiciones metafísicas y epistemológicas que se hacen en la empresa científica real, puede apreciar la ciencia como una actividad humana y humanizadora, y desmitificar el retrato de la ciencia como un coloso completamente objetivo y monolítico que inexorablemente produce verdad. Esta perspectiva realista es una base adecuada sobre la cual se pueden desarrollar de modo inteligente los temas de ciencia, tecnología y valores que cada vez son más críticos para la futura supervivencia de nuestra sociedad. Incluso si tales puntos no se pueden tratar en un curso de introducción a las ciencias, el alumno estará mejor preparado para tratar con ellos más adelante. Como mínimo, habrá un gran lastre de ideas falsas sobre la fiabilidad de la ciencia que no hará falta superar más tarde. Por lo tanto, el resto de este artículo deberá suponer la aceptación de algún valor para la Historia y la Filosofía de la ciencia en un curso de introducción a la Física. Aunque, ciertamente, no es mi intención sugerir que, de las tres metas mencionadas antes, ésta sea la más importante, sólo la que se descuida más a menudo. No propondría basar un curso de introducción a la Física únicamente en casos históricos. Se trata más bien de llamar la atención sobre un área problemática que necesita reajuste.

\section{DOS PROBLEMAS}

La dificultad más seria (Problema Uno) de exponer razonablemente a los alumnos la Historia y Filosofía de la ciencia en un curso de introducción a la 
Física es que tales cursos son, como es natural, impartidos normalmente por físicos. Además de ignorar en gran medida estos profesores aspectos de la Historia y filosofía de su disciplina, apenas toleran tratar esos asuntos o tienen para ellos poco uso, posiblemente como material anecdótico, adecuado sólo para los márgenes de un libro de texto. Esto es particularmente sorprendente, ya que aquéllos reconocidos universalmente como los gigantes del descubrimiento científico, (tales como Newton, Planck, Einstein, Schrodinger, Bohr, por nombrar unos pocos) se interesaron en puntos epistemológicos y filosóficos relacionados con los fundamentos de la Física. El sentimiento que muy a menudo se extiende entre los científicos en ejercicio es que tales intereses no son parte auténtica de la Física o que, aún peor, estos grandes hombres «malgastaron» parte de su tiempo y energía en tal esfuerzo. Para ellos la ciencia se ve como una fuente única y autónoma de conocimiento fiable. Habiendo dicho esto a modo de introducción, trataré ahora de los puntos específicos de mejora de los cursos: los libros de texto disponibles (Problema Dos). Es preciso tratar por separado aquéllos destinados a los estudiantes de ciencias y aquéllos para los que no las estudian.

La queja más corriente contra los textos de introducción a la Física para alumnos de ciencias es que abarcan demasiado, llegando a menudo a las mil cien páginas impresas o más (ver Cushing, 1984; French, 1984; Rigden, 1986, 1987). Se trata de demasiado material para que los alumnos lo dominen en dos o incluso tres semestres. La necesidad paranoica que se percibe de «cubrir toda la Física» en un curso introductorio obliga a los autores a incluir un gran número de temas típicos en cualquier manuscrito que pueda ser considerado en serio por un editor comercial para su publicación. Los editores creen, en gran medida de modo correcto (desgraciadamente), que la omisión de cualquiera de estas áreas molestará con certeza al número suficiente de usuarios potenciales (los profesores de Física indignados al encontrar un trato superficial de alguno de sus temas favoritos) para que las ventas se resientan. Estos libros de texto grandes y profusamente ilustrados son tan caros de producir y la competencia es tan fiera que debe venderse un número alto de textos los primeros años para rentabilizarlo, si se quiere mantener el precio dentro de límites razonables. Por lo tanto, los editores quieren manuscritos dirigidos a lo que ven más o menos como el grueso del mercado, sin lugar para un texto que se desvíe significativamente de esta norma. El resultado es un gran número de «clones» insípidos del texto de Física «típico».

Los textos de Física constituyen un caso peculiar entre los textos de introducción a la ciencia (si los comparamos con los de Química y Biología) en que estos enormes textos aparecen con material largamente desfasado, siendo el índice de contenidos y los puntos esenciales de presentación muy parecidos a como eran hace cincuenta o cien años (Holcomb, Resnick y Rigden, 1987). Hay muy poco espacio, cuando lo hay, dedicado a la Física «moderna» (un nombre seguramente inadecuado para los descubrimientos entre 1905 y 1925) de las teorías de la relatividad y cuántica. La Física se ve como un cuerpo esencialmente estático y verdades eternas que cambia sólo por acumulación. Esta dedicación a preservar y presentar toda verdad eterna genera un lastre de material sin posibilidades para un libro de texto o «biblia». Estamos condenados siempre a empezar con $T=0$ (Grosso modo, la mecánica newtoniana) para recitar en serie la grán épica de la ciencia. Naturalmente, dado un número fijo de clases, nos vemos incapaces de llegar al «fin» de la historia. Por tanto, el curso de introduc- 
ción termina dejando al alumno aún más lejos de comprender la situación de la Física como realmente se practica hoy día.

Dado este abarrotamiento serio del curso con material «duro» (a veces desfasado), no sorprende que pueda haber poco interés en cómo se originaron las grandes ideas de la Física y su desarrollo y que no se ponga énfasis en la relevancia de la Historia y la Filosofía de la ciencia para instructores y alumnos. También es paradógico que los libros de textos actuales muestran mentalidad de avestruz con respecto a las ilustraciones y aplicaciones modernas de «alta tecnología» contra las ilustraciones fosilizadas de «baja tecnología» usadas en aplicaciones de la más antigua Física clásica típica. Esto significa que los alumnos ven a menudo en televisión, o en encuentros cotidianos con equipos de fácil acceso, unos usos de principios físicos mucho más atractivos que los que encuentran en los laboratorios y demostraciones de su curso de Física. Esto, además de provocar el descenso del interés del alumno, crea el sentido de la irrelevancia para el mundo tecnológico moderno de la Física vista en clase. La Física como campo ya no interesa tanto a los alumnos del Instituto y Universidad como interesaba hace 20 o 30 años, y un factor que contribuye a ello es que la Física se percibe como menos importante para la sociedad del presente. La profesión de Física es responsable en gran medida de este estado de cosas, aunque ha habido físicos que durante décadas han levantado voces de insatisfacción con el contenido invariable de los cursos de introducción a la Física (Cushing, 1984; Holcomb, Resnick y Rigden, 1987; Rigden, 1986). Volveré más tarde con algunas sugerencias constructivas específicas, pero antes hablaré del estado de los cursos de introducción para alumnos que no son de ciencias.

Entre los libros de texto introductorios para alumnos que no son de ciencias hay una variedad más amplia que para los de ciencias superiores. Sin embargo, el nivel de presentación de la ciencia genuina es casi universalmente bajo. Se ha producido la defunción editorial de algunos libros buenos y exigentes, competentes técnicamente y que suponían un desafío intelectual, tales como $\mathrm{An} I n$ troduction to the Meaning and Structure of Science (Una introducción al significado y estructura de la ciencia), de Leon Cooper y The Nature of Physics (La naturaleza de la Física), de Peter Brancazio, cuya descatalogación ha sido permitida por sus editores. Muchos carecen virtualmente de matemáticas (algunos hasta de aritmética), y parecen tener el propósito de hacer entretenidos los cursos optativos. No hay una mezcla equilibrada entre una Historia y Filosofía de la ciencia competentes (no sólo anecdóticas) y la Física real. Debe recalcarse, por supuesto, la naturaleza factual y explicativa de la ciencia como en el excelente Physics for the Inquiring Mind (Física para la mente inquisitiva), de Eric Rogers. Pero eso no es todo, la media deplorable de cursos de Física para alumnos que no son de ciencias es en parte resultado del bajo nivel de matemáticas que se pide ahora en general al alumno en nuestros Institutos y del pequeño número de cursos de ciencias puras requeridos para el título universitario general.

Una editorial importante (Cambridge University Press) se ha comprometido recientemente a producir un libro de texto general de introducción a la Física, The Mechanical Universe (El universo mecánico) de Olenick, Apostal, y Goodstein. No se han escatimado gastos en publicar además del propio libro un número extenso de películas acompañando al texto. Los autores afirman que el libro se dirige a alumnos con sólo una base de álgebra y trigonometría. Desgraciadamente el producto resulta tener un ritmo y un nivel demasiado altos, como el artículo crítico de Aron (1986) documenta con doloroso detalle. Que 
este proyecto haya fracasado tan estrepitosamente es especialmente descorazonador porque los editores comerciales siempre han tenido dudas en acometer empresas de este tipo. Puede hacer que otros editores sean más recelosos en el futuro. Aunque también Cambridge ha publicado recientemente From Paradox to Reality (De la paradoja a la realidad), de Fritz Rohrlich, un libro adecuado para el curso optativo general de un semestre. Es una señal esperanzadora.

Una meta razonable y asequible para cualquier curso de introducción a la Física, sea para alumnos de ciencias o no, es estructurar la elección y presentación del material de modo que la mayor parte del segundo semestre se dedique a la relatividad y a la física cuántica (la teoría del caos también es suceptible de presentación elemental usando modelos simples a modo de ilustración). Una justificación evidente para el empleo de partes de artículos y textós clásicos en ese libro es que a menudo tienen mayor claridad que los tratamientos dados en algunos libros de textos modernos. Los textos clásicos también dan al alumno cierto sentido de la historia del descubrimiento en la ciencia. Al terminar cualquier curso de introducción a la Física, un alumno debería tener una apreciación sólida del cambio radical de la naturaleza de las explicaciones requirido por la llegada de la mecánica cuántica. La explicación causal, al nivel fundamental de los fenómenos físicos, ya no existe. Esta es la lección más profunda de la Física del siglo XX para el entendimiento del mundo real. Un desarrollo de los teoremas de Bell, cuyos resultados experimentales eliminan por principio cualquier visión localista y determinista del mundo, entra bien dentro de la capacidad técnica de un alumno de Física General. Normalmente los presento en mis clases de primer curso, sea de una carrera de ciencias o no. Una apreciación de este cambio profundo de nuestra visión metafísica del mundo producida por resultados empíricos en Física ilustra concreta e irrefutablemente las conexiones íntimas entre Filosofía y ciencia.

\section{QUE HACER}

Es más fácil empezar por los cambios necesarios para los cursos que no son principalmente de ciencias porque los físicos no tienen interés excesivo en los contenidos de tales cursos en comparación con los programas de los suyos propios. Los mayores obstáculos a la hora de enseñar cursos adecuados a alumnos que no son de ciencias son que los profesores de Física no se han interesado por estos cursos en particular y que, aunque se les asignara esa tarea, no son expertos en Historia y Filosofía de la ciencia. La necesidad altamente práctica de producir más horas de crédito para alumnos en las facultades de Física ha contribuido a superar el desdén mostrado hacia los cursos en carreras que no sean de ciencia. También ha dado oportunidad, desgraciadamente, a la aparición de unos cuantos libros malos y repulsivos «de entretenimiento general».

Si los cursos de introducción a la Física, tanto para los que estudian ciencias como para los que no, pueden centrarse en la relatividad y la física cuántica a fin de arrastrar la instrucción de la Física hasta la última década del siglo XX, la estructura rígida de los cursos de ciencias, hasta los más especializados, se romperá y será posible un cambio significativo dentro del nuevo ambiente. Más aún, una instrucción con un cierto sentido histórico producirá con suavidad los cambios dramáticos en las visiones del mundo que siempre han acompañado a los avances en la ciencia. Desde la mecanicista, de Aristóteles a Newton, has- 
ta la indeterminista cuántica. Se verá que la localización de este cambio corrector no se encontrará aislada del pasado histórico distante, sino que será una parte dramática de la empresa científica actual. Por una parte, esto puede ayudar a producir un grupo bien informado de ciudadanos normales que posean cierta comprensión de los principios de la ciencia y una imagen realista de la construcción del conocimiento científico. Por otra parte, puede ayudar a formar un cuerpo de jóvenes científicos que aprecien que la ciencia tiene profundas implicaciones filosóficas para la visión que el hombre tiene del mundo y que la generación del conocimiento científico tiene similitudes importantes con actividades en otros campos. El vacío entre las «dos culturas» se alimenta más de la ignorancia mutua que de cualquier diferencia que exista obtenible por las «ciencias» contra las «artes». Una apreciación de este hecho puede ser la lección general más importante que se haga comprender a los alumnos mediante un nuevo acercamiento a la enseñanza científica.

Parte de la hostilidad intrínseca de muchos científicos hacia desarrollos históricos y filosóficos de la naturaleza de la ciencia (los cuales creen conocer con bastante claridad) puede ser superada por nuevos libros de texto que consigan presentar la Física enfocada en teorías y desarrollos modernos sobre una base histórica adecuada. Debemos romper el círculo vicioso en el que los editores comerciales perciben un mercado que no tolerará ningún libro fuera de la norma y en el que aquellos profesores que puedan querer experimentar algo nuevo quedan bloqueados por la carencia de textos adecuados. (Sólo queda la opción de hacer el enorme trabajo de base necesario para escribir un texto muy diferente, aunque sin tener virtualmente ninguna expectativa de logar publicarlo.) Sin embargo, la carga de la responsabilidad de producir condiciones que lleven a este cambio recae principalmente en la profesión de la Física, no en los editores.

En el pasado, la comunidad académica de Física, junto con la mayoría de la empresa educativa de este país, ha eludido su responsabilidad educativa al no requerir cursos de ciencias más exigentes para todos los alumnos universitarios, con un desinterés casi activo y ciertamente elitista en comunicar al público en general cualquier comprensión genuina de la Física moderna, permaneciendo rígidos en su acercamiento a la enseñanza introductoria a la Física para alumnos especializados aunque el número de éstos ha disminuido, y por su arrogancia a la hora de ignorar los orígenes históricos y filosóficos de la Física y la relevancia continua de estas disciplinas dentro de la ciencia moderna. Es necesario un mayor trabajo interdisciplinar por parte de los científicos en nuestra propia prensa científica especializada para remediar la situación presente. Debemos aprovechar la oportunidad para el cambio que nos presenta la actual insatisfacción con los libros de texto para alumnos de ciencias e ingeniería (ver Holcomb, Resnick y Rigden, 1987). Si bien es útil la discusión en publicaciones filosóficas y educativas tales como Interchange (intercambio), es esencial que los propios físicos también participen activamente en cambiar el clima de su disciplina. Entonces, tal vez, los editores comerciales lanzarán de verdad los globos sonda que puedan anunciar una estructuración genuina de la enseñanza de la introducción a la Física en los Estados Unidos. Hasta ahora, Cambridge University Press parece ser la única editorial dispuesta a tal compromiso. Es particularmente irónico que este problema serio que existe en los Estados Unidos sea atacado por una editorial cuya sede está en Inglaterra. ¡Deberíamos ocuparnos de nuestros propios pacientes! 


\section{Referencias}

Arons, A. B. (1986). Review of The Mechanical Universe: Introduction to Mechanics and Heat. American Journal of Pbysics, 54, 857-862.

Cushing, J. T. (1984). But, who will publish a sane introductory physics tex? American Joumal of Physics, 52, 1069-1070.

French A. P. (1984). Post-use review: Pbysics for Science and Engineering, Pant I. American Joumal of Physics, 52.

Holcomb, D. F., Resnick, R. y Rigden, J. S. (1987, mayo). New approaches to introductory physics. Pbysics Today, p. 87.

Rigden, J. S. (1986). The current challenge: Introductory physics. American Joumal of Physics, 54.

Rigden, J. S. (1987). Introductory physics and the burden of truth. American Joumal of Physics, 55.

\section{Una tarea difícil. Historia, filosofía e introducción}

a la física. James T. Cusbing. CL\&E, 1991, 11-12, pp. 181-187

Datos sobre el autor: James T. Cushing es profesor de física en la Universidad de Notre Dame.

Dirección: Universidad de Notre Dame. Departamento de Física. Notre Dame, Indiana. 46556 USA.

Artículo original: A Tough Act-History, Philosophy, and Introductory Physics (An American Perspective). En Interchange, 1989; Vol. 20, n. ${ }^{\circ} 2$; pp. 54-59. Reproducido con autorización. Traducción de Eduardo Braun.

(C) De todos los artículos. Deberá solicitarse por escrito autorización de CL\&E y de los autores para el uso en forma de facsímil, fotocopia o cualquier otro medio de reproducción impresa. CL\&E se reserva el derecho de interponer las acciones legales necesarias en aquellos casos en que se contravenga la ley de derechos de autor. 